\title{
Special Issue: Recent Advances on Control and Architectural Design of Robotic Systems
}

\author{
Krzysztof Kozłowski
}

Published online: 14 February 2015

(C) The Author(s) 2015. This article is published with open access at SpringerLink.com

This Special Issue entitled "Recent Advances on Control and Architectural Design of Robotic Systems" is devoted to current research activities on the above topic that was presented during the Twelve National Conference on Robotics held in Świeradów - Zdrój, Poland, September 12-16, 2012. Members of The Programme Committee selected 8 papers based on their merits and quality of presentation during the conference and asked their authors to prepare extended versions for possible publication at the Journal of Intelligent and Robotic Systems. As a result of the reviewing procedure, six papers were finally accepted for publication at the Journal of Intelligent and Robotic Systems. They are listed below.

1. Maciej M. Michałek, Marcin Kiełczewski, Cascaded VFO control for non-standard N-trailer robots,

2. Alicja Mazur, Mateusz Cholewiński, Virtual force concept in steering mobile manipulators with skid - steering platform moving in unknown environment,

3. Adam Ratajczak, Krzysztof Tchoń, Parametric and non-parametric Jacobian motion planning for non - holonomic robotic systems,

K. Kozłowski $(\square)$

Chair of Control and Systems Engineering,

Poznań University of Technology, Poznań, Poland

e-mail: krzysztof.kozlowski@put.poznan.pl
4. Dariusz Pazderski, Dorota K. Waśkowicz, Krzysztof Kozłowski, Motion control of vehicles with trailers using transverse function approach controller properties analysis,

5. Joanna Płaskonka, Different kinematic pathfollowing controllers for a wheeled mobile robot of $(2,0)$ type,

6. Tomasz Kornuta, Cezary Zieliński, Robot control system design exemplified by multi-camera visual servoing.

Paper no. 1 presents cascaded Vector-FieldOrientation (VFO) control strategy as a unified solution to the set-point and trajectory tracking control tasks for articulated robots equipped with arbitrary number of passively off-axle hitched trailers (in general N-Trailer). Control input limitations of the robot are directly taken into account by utilization of a simple velocity scaling procedure that preserves an instantaneous motion curvature of a tractor. Description and theoretical substantiation of the concept are followed by the results of experimental validation tests conducted with usage of a 3-trailer semi-autonomous vehicle.

Paper no. 2 solves control problem for mobile manipulator mounted on skid-steering platform. As a control scheme the concept of virtual force is presented and its mathematical proof is discussed using Lyapunov-like function. The equation of the implicit function generates the reference signals needed in control algorithm. Efficiency and robustness of 
control scheme to parametric uncertainty of terrain, on that the mobile manipulator is moving, are illustrated by numerous simulations.

In paper no. 3 authors discuss computational aspects of the Jacobian motion planning algorithms for non-holonomic robotic systems. Jacobian motion planning algorithms are derived by means of the continuation (homotopy) method applied to the inverse kinematics problem in the space of control functions. Two methods of representing the control functions are studied: parametric and non-parametric. The parametric method parametrizes the control functions by truncated orthogonal series. The paper focuses on the non-parametric Jacobian pseudo inverse motion planning algorithms incorporating a higher order integration method. Performance of this algorithm is illustrated by the numeric solution of motion planning problem for the rolling ball kinematics, and compared with the performance of its parametric version.

Paper no. 4 is concentrated on analysis of the control solution using the transverse function approach. The controller with its properties considered in the paper is designed for a non - holonomic vehicle with on-axle passive trailers. The main problem investigated is the optimal parametrization of the transverse functions in order to ensure low sensitivity to the measurement noise and high tracking accuracy. Theoretical analysis referring to transverse function scaling using dilation is illustrated by results of extensive numerical simulations.

In paper no. 5 a comparative study of selected kinematic path following controllers for a wheeled mobile robot of $(2,0)$ type is presented. The control strategies are based on one of two approaches to the path parameterization - either the Serret-Frenet frame with an orthogonal projection or the Serret-Frenet frame with a non-orthogonal projection of a robot on the desired path. The complete control system for the wheeled mobile robot consists of two parts, a kinematic controller and a dynamic controller. Theoretical derivations are illustrated through computer simulations.

In paper no. 6 authors investigate the problem of designing robot control architecture systems based on the concept of an embodied agent. Multi-level decomposition of system structure enables the formulation of the operation of each of the resulting subsystems in terms of transition functions, specifying their state evolution. The paper also introduces mathematical and graphical notations, complementing each other, defining formally the control system behaviour. As an example the specification of a control system of a robot utilizing multi- camera visual servoing for object following is presented.

I strongly believe that these papers due to new theoretical and experimental results in robot control would be of interest to the Readers of the Journal of Intelligent and Robotic Systems.

I would like to express my appreciation to the authors of this Special Issue for the high quality of their work and the cooperation during the review process. Also, I would like to thank all reviewers for their valuable comments and suggestions that contributed significantly to the improvement of the papers quality. Special thanks are directed to Professor Kimon V. Valavanis, Editor-in-Chief of the Journal of Intelligent and Robotic Systems giving me the opportunity of publishing this Special Issue.

Open Access This article is distributed under the terms of the Creative Commons Attribution License which permits any use, distribution, and reproduction in any medium, provided the original author(s) and the source are credited. 